\title{
Time course-changes in phosphatidylcholine profile during oxidative modification of low-density lipoprotein
}

Naoko Sasabe ${ }^{1 \dagger}$, Yuka Keyamura ${ }^{1,2 \dagger}$, Takashi Obama ${ }^{1}$, Nozomi Inoue ${ }^{1}$, Yukihiro Masuko ${ }^{1}$, Yu Igarashi ${ }^{1}$, Toshihiro Aiuchi ${ }^{1}$, Rina Kato ${ }^{1}$, Tomohiro Yamaguchi ${ }^{1}$, Hiroshi Kuwata ${ }^{3}$, Sanju Iwamoto ${ }^{4}$, Akira Miyazaki ${ }^{4}$, Shuntaro Hara ${ }^{3}$, Tomohiro Yoshikawa ${ }^{2}$ and Hiroyuki Itabe

\begin{abstract}
Background: Oxidized phosphatidylcholines (oxPC) and lysophosphatidylcholine (lysoPC) generated during the formation of oxidized low-density lipoprotein (oxLDL) are involved in atherosclerotic lesion development. We investigated the time course-changes in phosphatidylcholine (PC) molecular species during oxidation of LDL to determine how those atherogenic PCs are produced.
\end{abstract}

Methods: Human and rabbit LDLs were pretreated with or without a selective platelet-activating factor acetylhydrolase (PAF-AH) inhibitor. LDL was oxidized by incubation with copper sulfate, and PC profiles were analyzed by liquid chromatography-tandem mass spectrometry.

Results: When human LDL was oxidized, the peak areas for polyunsaturated fatty acid (PUFA)-containing PC species dramatically decreased after a short lag period, concomitantly lysoPC species increased sharply. Although a variety of oxPC species containing oxidized fatty acyl groups or cleaved acyl chains are formed during LDL oxidation, only a few oxPC products accumulated in oxLDL: 1-palmitoyl-2-(9-oxo-nonanoyl) PC and long-chain oxPC with two double bonds. Pretreatment of LDL with the PAF-AH inhibitor greatly reduced lysoPC production while it had no effect on lipid peroxidation reactions and OxPC profiles. Rabbit LDL, which has a different composition of PC molecular species and needs a longer time to reach achieve full oxidation than human LDL, also accumulated lysoPC during oxidation. The increase in lysoPC in rabbit oxLDL was suppressed by pretreatment with the PAF-AH inhibitor. The major oxPC species formed in rabbit oxLDL were almost the same as human oxLDL.

Conclusions: These results suggest that lysoPC species are the major products and PAF-AH activity is crucial for lysoPC generation during oxidation of LDL. The OXPC species accumulated are limited when LDL is oxidized with copper ion in vitro.

Keywords: Oxidized LDL, PC molecular species, LC-MS/MS, Oxidized PC, PAF-AH, Pefabloc, lysoPC, PONPC, Rabbit LDL

\footnotetext{
*Correspondence: h-itabe@pharm.showa-u.ac.jp

${ }^{\dagger}$ Equal contributors

'Division of Biological Chemistry, Department of Molecular Biology, Showa University School of Pharmacy, Tokyo, Japan

Full list of author information is available at the end of the article
} 


\section{Background}

Oxidative modification of low-density lipoprotein (LDL) is involved in atherosclerotic lesion development [1-3]. Studies have shown significant increases in plasma circulating oxidized LDL (oxLDL) in patients with cardiovascular diseases [4-8]. LDL consists of phospholipids, cholesterol ester, triacylglycerol, and an apolipoprotein B (apoB) together with several minor constituents, so that oxLDL may contain a large variety of oxidized products and modified molecules [9-11].

Many biological and pathological activities of oxLDL have been investigated, and oxidized phosphatidylcholine (oxPC) and lysophosphatidylcholine (lysoPC) formed during oxidation of LDL are thought to be responsible for some oxLDL functions. OxPC is a mixture of diverse products characterized by various functional groups, including both long chain acyl groups with hydroperoxide or hydroxyl function and truncated short chain acyl groups. Macrophage scavenger receptors take up oxLDL leading to foam cell formation, where oxPC is involved in recognition by scavenger receptors [12,13]. OxPC could act as a peroxisome proliferator activating receptor (PPAR) $-\gamma$ activator to induce inflammatory and metabolic responses [14]. It promotes chemokine production in endothelial cells $[15,16]$ and proliferation and calcification of smooth muscle cells [17]. In addition, oxPC induces inflammatory reactions by acting as an environmental pathogen mimetic [18]. OxPC, especially PC hydroperoxides $(\mathrm{PCOOH})$, increases macrophage adhesion to ICAM-1 through reorganization of microfilaments [19]. LysoPC is another atherogenic metabolite formed in oxLDL that shows many biological effects including attenuation of endothelial functions and proliferation of smooth muscle cells $[20,21]$.

OxPC is further modified chemically or enzymatically in plasma. Several enzymes metabolize oxPC, including phospholipid hydroperoxide glutathione peroxidase (PHGPx) [22], secretory phospholipase $\mathrm{A}_{2}$ type IIA (sPLA ${ }_{2}$-IIA) [23], lecithin-cholesterol acyltransferase (LCAT) [24], and platelet-activating factor acetyl hydrolase (PAF-AH/Lp-PLA ${ }_{2}$ ) [25]. These enzyme activities make oxidized $\mathrm{PC}$ profiles more complex.

PAF-AH is a unique plasma phospholipase that hydrolyzes PC species containing short and/or hydrophilic acyl chains as substrates in addition to PAF itself $[25,26]$. It is considered that PAF-AH contributes substantially to the detoxification of pro-atherogenic oxPC products into lysoPC. Steinbrecher reported that the phospholipase $\mathrm{A}_{2}$ activity for short-chain PC substrates in human LDL was not separated from PAF-AH [27]. The enzyme associates with lipoprotein particles, LDL and high-density lipoprotein (HDL), in human plasma [25] and is found primarily in association with HDL in rabbit and rodent plasma $[28,29]$. Because PAF-AH has an active serine residue in its catalytic center, enzyme activity is inhibited by serineprotease inhibitors such as phenylmethylsulfonyl fluoride (PMSF). Pefabloc, a sufonylfluoride compound similar to PMSF, does not inhibit paraoxonase-1, LCAT, or venom phospholipase $A_{2}$, has been used as a selective inhibitor of PAF-AH [30-32].

Although the pathological impact of oxLDL is recognized, neither the complete structure of oxLDL nor the molecular process of oxLDL generation has been fully determined. Recently liquid chromatography-tandem mass spectrometry (LC-MS/MS) has been applied to the comprehensive analysis PC molecular species including oxidized products [33-35]. Rabbits have been used for diet-induced atherosclerosis experiments, although many animals including rodents are not susceptible to atherosclerosis without genetic manipulation. Checking PC profiles of human and rabbit oxLDLs could give an idea for atherogenic oxPC products. In the present study, we investigated the PC profile changes during copperinduced oxidation of LDL to elucidate the process of PC modification by LC-MS/MS. We also investigated the contribution of PAF-AH to $\mathrm{PC}$ profile changes using a PAF-AH inhibitor and rabbit LDL in addition to human LDL.

\section{Results}

\section{PC profile change in human LDL during oxidation}

Human LDL treated with or without pefabloc, a PAF-AH inhibitor, was incubated with copper sulfate for up to $3 \mathrm{~h}$ and the profile of PC molecular species was determined. When human LDL was pretreated with pefabloc for $30 \mathrm{~min}, 98 \%$ of the PAF-AH activity was inhibited, as determined using 2-thio-PAF as substrate. Pretreatment of LDL with pefabloc showed no differences in increases of thiobarbituric acid-reactive substances (TBARS) and electromobility shift in agarose gel electrophoresis during oxidation (Figure 1), suggesting that peroxidation reactions in the LDL proceed equally in pefabloc-treated LDL and nontreated LDL.

Using LC-MS/MS with multiple-reaction monitoring (MRM), 42 PC species with selected $\mathrm{m} / \mathrm{z}$ values were detected simultaneously in each oxLDL sample (Table 1). These PC species were classified into five groups: PC containing polyunsaturated fatty acids (PUFA-PC), PC with only saturated or monounsaturated fatty acids (S/MUFA-PC), lysoPC, oxPC with truncated acyl chains (cleaved oxPC), and oxPC with oxidized acyl chains (long chain oxPC). Figure 2 shows the changes in peak area of each $\mathrm{PC}$ species during copper-induced oxidation of human LDL. PUFA-PC species did not change for the first $30 \mathrm{~min}$, then decreased dramatically (Figure 2A), whereas S/MUFA-PC did not change throughout the incubation (Figure 2B). LysoPC species, primarily 16:0LPC and 18:0-LPC, increased gradually until $30 \mathrm{~min}$ and 


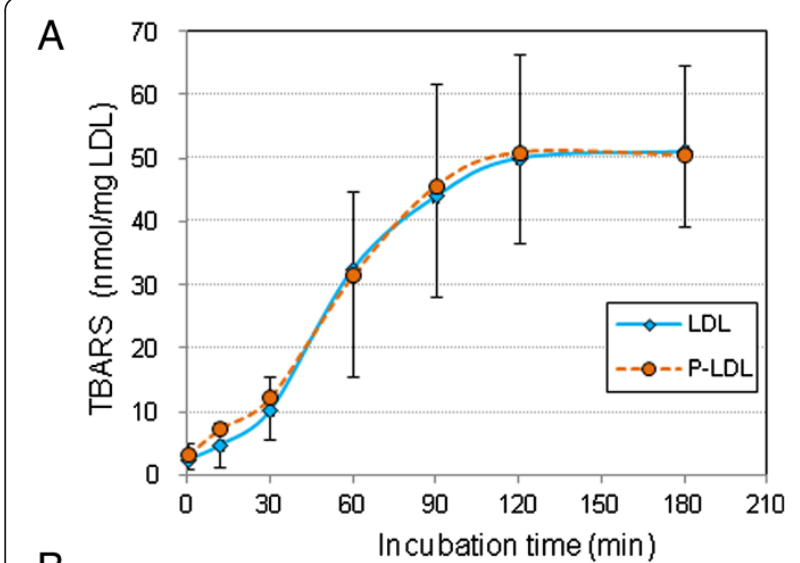

B

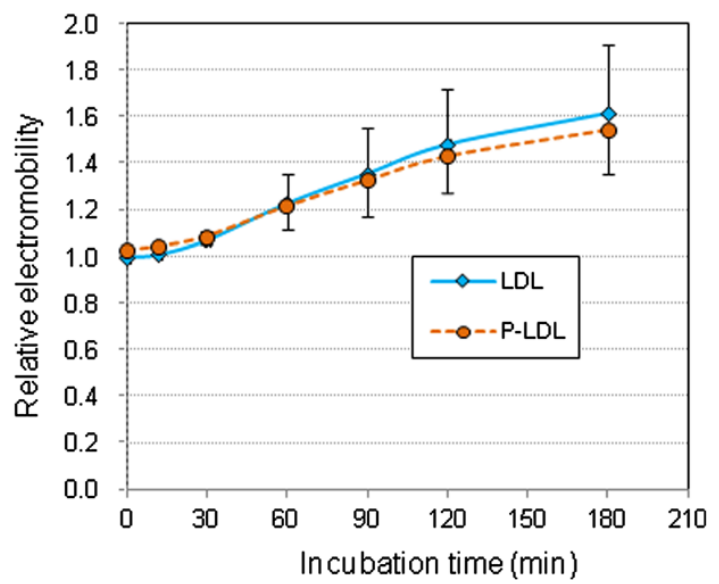

Figure 1 Time course of copper-induced oxidation of human LDL. Human LDL $(0.2 \mathrm{mg} / \mathrm{mL})$ treated either with or without a PAF-AH inhibitor, pefabloc, was incubated with copper sulfate $(5 \mu \mathrm{M})$ at $37^{\circ} \mathrm{C}$ for up to $3 \mathrm{~h}$. The oxidative change of $L D L$ during the incubation was evaluated by production of TBARS (A) and relative mobility on agarose gel electrophoresis (B). Values indicated are mean \pm SD of 8-10 experiments.

then jumped up during copper-induce oxidation (Figure 2C). The amounts of oxPC increased slightly throughout the incubation period. Many oxPC species were formed, but the major product appeared to be 1palmitoyl-2-(9-oxo-nonanoyl) PC (PONPC; $\mathrm{m} / \mathrm{z}=650.6$ ) (Figure 2D). Among the long-chain oxPC, species with two double bonds, derived from linoleate-containing PCs, accumulated during the incubation period. In contrast, those with four double bonds, likely derived from arachidonate-containing PCs, did not accumulate or even decreased after $1 \mathrm{~h}$ incubation (Figure 2E).

Figure 3 shows the PC profile changes during the oxidation of pefabloc treated-human LDL. After the lag time, PUFA-PC species began to decrease at $60 \mathrm{~min}$ and S/ MUFA-PC did not change throughout the incubation (Figure 3A, B). LysoPC species increased during the incubation, although the peak areas were much smaller than those without pefabloc treatment (Figure $3 \mathrm{C}$ ). The major oxPC species accumulated were PONPC and long-chain oxPC species with two double bonds (Figure 3D, E) that were also the major species in the absence of pefabloc treatment.

We calculated the increase or decrease of peak area obtained after the $3 \mathrm{~h}$ incubation period for each species to see the effect of pefabloc on the PC profiles (Figure 4). All of the PUFA-PC reduced their peak areas, showing that inhibition of PAF-AH had little effect on changes in PUFAPC following treatment with copper sulfate (Figure 4A). LysoPC increased their peak areas during the oxidation reaction, and the increase of lysoPC was greatly suppressed when PAF-AH was inhibited (Figure 4C). Pefabloc treatment had limited effect on the increase of cleaved oxPC and long-chain oxPC species, while cleaved oxPC species with five carbon-acyl chains $(\mathrm{m} / \mathrm{z}=594.2,610.6$ and 622.6) increased slightly (Figure 4D, E).

\section{PC profiles in rabbit LDL during oxidation}

Next, we examined changes in the PC profile in rabbit LDL during oxidation. LDL was prepared from New Zealand white rabbits fed normal diet supplemented with $0.5 \%$ cholesterol to increase LDL content in plasma. The PC profile of rabbit LDL is substantially different from that of human LDL. Rabbit LDL is relatively rich S/MUFA-PC and lysoPC. Rabbit LDL was needed a longer incubation than human LDL to reach the maximum oxidation, which may be caused by lower content of PC species with more than five double bonds. PAF-AH activity in the rabbit LDL fraction was estimated to be $2.9 \mathrm{nmol} / \mathrm{min} / \mathrm{mg}$, which is $25 \%$ of that in human LDL. The PAF-AH activity in the LDL fraction was $6.0 \%$ of HDL fraction recovered from the same rabbits, which corresponds to a previous report that PAF-AH is present mostly in HDL in rabbit plasma and very little in LDL [28]. Pretreatment of the rabbit LDL fraction with pefabloc reduced PAF-AH activity by $91 \%$.

Rabbit LDL, with or without pefabloc treatment, was incubated with copper sulfate for up to $8 \mathrm{~h}$, and the profiles of PC molecular species were determined. Figures 5 and 6 show the time-course changes in peak area of each PC molecular species in rabbit oxLDL. The profile of S/MUFA-PC did not change throughout the incubation, and PUFA-PC appeared to decrease after $1 \mathrm{~h}$ (Figures 5A, 5B, 6A, and 6B). LysoPC increased during oxidation of rabbit LDL but it did not when rabbit LDL was pretreated with PAF-AH inhibitor (Figures 5C, 6C). These results, together with those from human oxLDL, indicate that the contribution of PAF-AH is required for lysoPC formation during oxidative modification of LDL. Many of the oxPC species increased their peak areas during the oxidation, however the major species accumulated in rabbit oxLDL, PONPC and long-chain oxPC with two 
Table 1 List of 42 PC species monitored by LC-MS/MS

\begin{tabular}{|c|c|c|c|}
\hline $\mathrm{m} / \mathrm{z}$ & Molecular species & $\mathrm{m} / \mathbf{z}$ & Molecular species \\
\hline S/MUFA-PC & & Long chain oxPC & \\
\hline 732.6 & $32: 1$ & 772.6 & $34: 2+14$ \\
\hline 734.6 & $32: 0$ & 774.6 & $34: 2+16$ \\
\hline 760.6 & $34: 1^{*}$ & 790.6 & $34: 2+32$ \\
\hline 788.6 & $36: 1$ & 796.6 & $36: 4+14$ \\
\hline 790.6 & $36: 0$ & 798.6 & $36: 4+16$ \\
\hline \multirow[t]{2}{*}{678.4} & $28: 0^{*}$ & 814.6 & $36: 4+32$ \\
\hline & & 800.6 & $36: 2+14$ \\
\hline PUFA-PC & & 802.6 & $36: 2+16$ \\
\hline 756.6 & $34: 3$ & 818.6 & $36: 2+32$ \\
\hline 758.6 & $34: 2^{*}$ & 824.6 & $38: 4+14$ \\
\hline 780.6 & $36: 5$ & 826.6 & $38: 4+16$ \\
\hline 782.6 & $36: 4^{*}$ & 842.6 & $38: 4+32$ \\
\hline 784.6 & $36: 3$ & & \\
\hline 786.6 & $36: 2$ & Cleaved oxPC & \\
\hline 806.6 & $38: 6$ & 594.6 & 1-palmitoyl-2-(5-oxovaleroyl) PC* \\
\hline 808.6 & $38: 5$ & 610.6 & 1-palmitoyl-2-glutaroyl PC* \\
\hline 810.6 & $38: 4$ & 622.6 & 1-stearoyl-2-(5-oxovaleroyl) PC \\
\hline \multirow[t]{2}{*}{834.6} & $40: 6$ & 638.6 & 1-stearoyl-2-glutaroyl PC \\
\hline & & 650.6 & 1-palmitoyl-2-(9oxo-nonanoyl)PC* \\
\hline LysoPC & & 664.6 & 1-palmitoyl-2-(5-oxo-octenoyl) PC \\
\hline 496.2 & 16:0-lysoPC* & 666.6 & 1-palmitoyl-2-azelaoyl PC* \\
\hline 524.2 & 18:0-lysoPC & 692.6 & 1-stearoyl-2-(5-oxo-octenoyl) PC \\
\hline 494.2 & 16:1-lysoPC & 706.6 & 1-stearoyl-2-(6-oxo-nonenoyl) PC \\
\hline 522.2 & 18:1-lysoPC & $652.6 a$ & 1-O-hexadecyl-2-azelaoyl PC* \\
\hline
\end{tabular}

In MRM mode, signals corresponding to selected $\mathrm{m} / \mathrm{z}$ values with a fragment ion of 184 were selectively detected. *PC species identified by comparison with commercially available synthetic standard PC species. The other species are inferred from MS data available in the literature.

double bonds, were the same as those in human oxLDL (Figures 5D, 5E, 6D and 6E).

Figure 7 shows the change of peak area for each species after the $8 \mathrm{~h}$ incubation of rabbit LDL with or without pefabloc treatment. It was observed again that pretreatment of LDL with pefabloc had no effect on the behavior of PUFA-PC and S/MUFA-PC during the oxidation of rabbit LDL, which is very similar to human LDL (Figure 7A, B). LysoPC increased their peak areas during the oxidation reaction, and the lysoPC formation was suppressed in pefabloc-treated LDL (Figure 7C). Pefabloc treatment had enhanced the accumulation of cleaved oxPC species and suppressed long-chain oxPC species with two double bonds (Figure 7D, E).

\section{Protein modification with oxPC}

During oxidation of LDL, apoB-oxPC adducts are formed and can be detected by sandwich ELISA using antibodies recognizing oxPC and apoB [36]. Inhibition of PAF-AH activity in human LDL increased apoB-oxPC adducts formed in oxLDL, as judged from immunoreactivity in sandwich ELISA (Figure 8). PAF-AH hydrolyzes oxPC species with short and hydrophilic acyl chains including chemically reactive aldehyde derivatives [26]. We accordingly speculate that PAF-AH is protective to apoB-oxPC adduct formation.

\section{Discussion}

OxLDL is thought to be an important factor in atherosclerosis. Evidence is accumulating that the plasma level of in vivo oxLDL is a predictor of cardiovascular disease [4-8]. Previous studies revealed that many atherogenic oxPCs and lysoPC are formed in oxLDL $[12,15,18,20]$. However, the manner in which LDL is oxidatively modified remains to be determined. In this study we analyzed the time-course changes in the PC profile during LDL oxidation using LC$\mathrm{MS} / \mathrm{MS}$ to view the full picture of oxPC generation and the contribution of PAF-AH. Our study shows that PAF-AH has a crucial role in generating lysoPC during LDL oxidation. Many cleaved oxPC and long-chain oxPC species are 

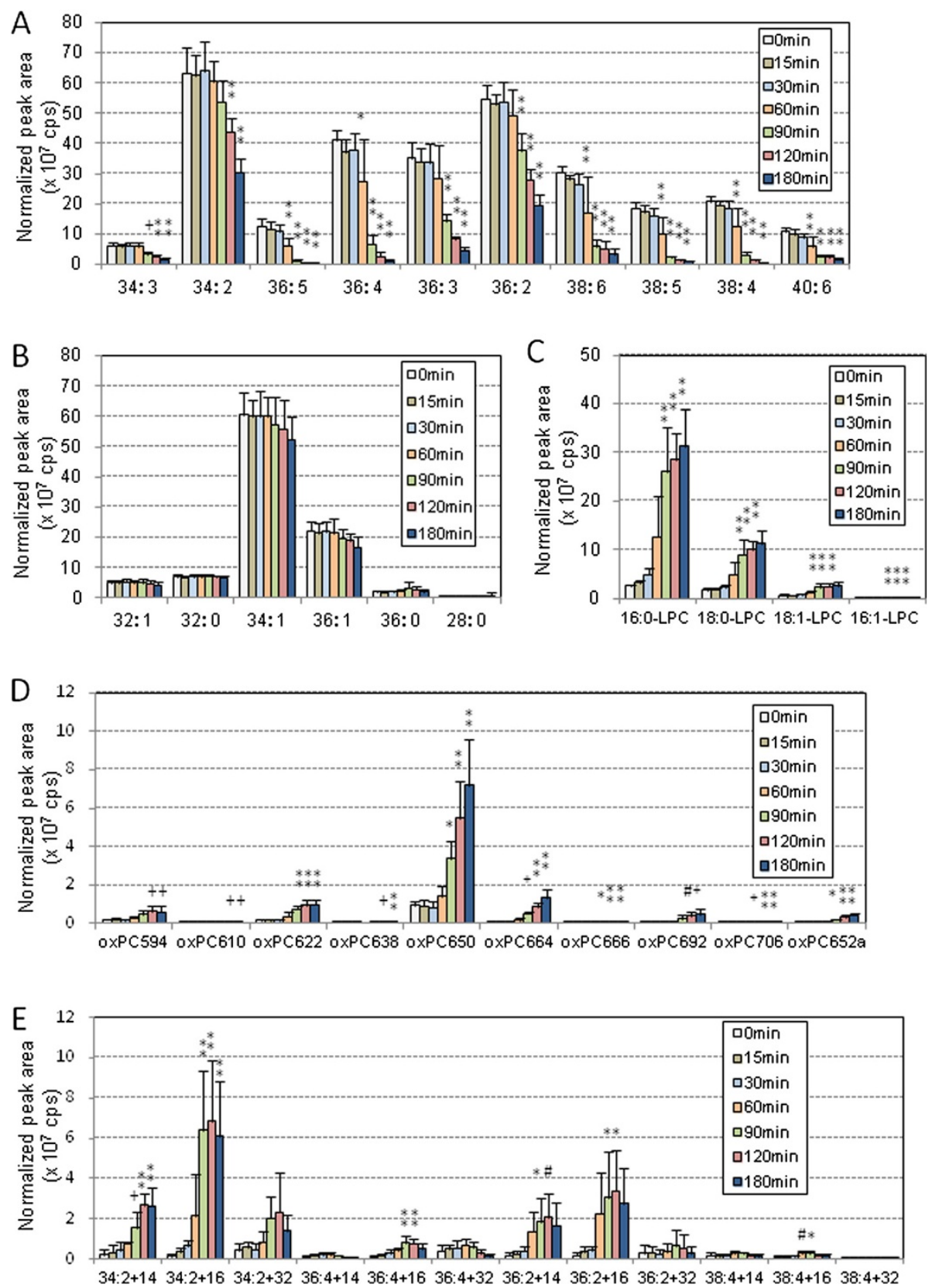

Figure 2 PC profile changes during copper-induced oxidation of human LDL. Human LDL $(0.2 \mathrm{mg} / \mathrm{mL})$ was incubated with copper sulfate $(5 \mu \mathrm{M})$ at $37^{\circ} \mathrm{C}$ for up to $3 \mathrm{~h}$. Lipids were extracted by the Bligh and Dyer method from $10 \mu \mathrm{g}$ of oxLDL after addition of 20 pmol of didecanoyl PC as internal standard. Total lipid extract was subjected to LC-MS/MS analysis to detect 42 PC and modified PC molecular species by MRM mode, and they were classified into five categories: PUFA-PC (A), S/MUFA-PC (B), lysoPC (C), cleaved-chain oxPC (D) and long-chain oxPC (E). The peak area (cps) for each species was normalized based on the peak areas of internal standard, and the means and SD were calculated from six independent preparations. Statistical significance of comparison with the sample without incubation ( 0 min) was calculated by ANOVA; *, $p<0.05$; $\#, p<0.01 ;+, p<0.005 ; * *, p<0.001$.

generated, but the data show only a few major oxPC species accumulating in oxLDL.

By simultaneously and selectively detecting 42 PC species in a sample using MRM mode of LC-MS/MS, we followed changes in PC profiles during copper-induced oxidation.
Because standards for all of the PC species were not available, absolute quantitation of the $\mathrm{PC}$ profiles cannot be completed. The data are shown as the relative peak areas of the PC species after normalizing on the basis of internal standard. As expected, most of the PUFA-PC species 

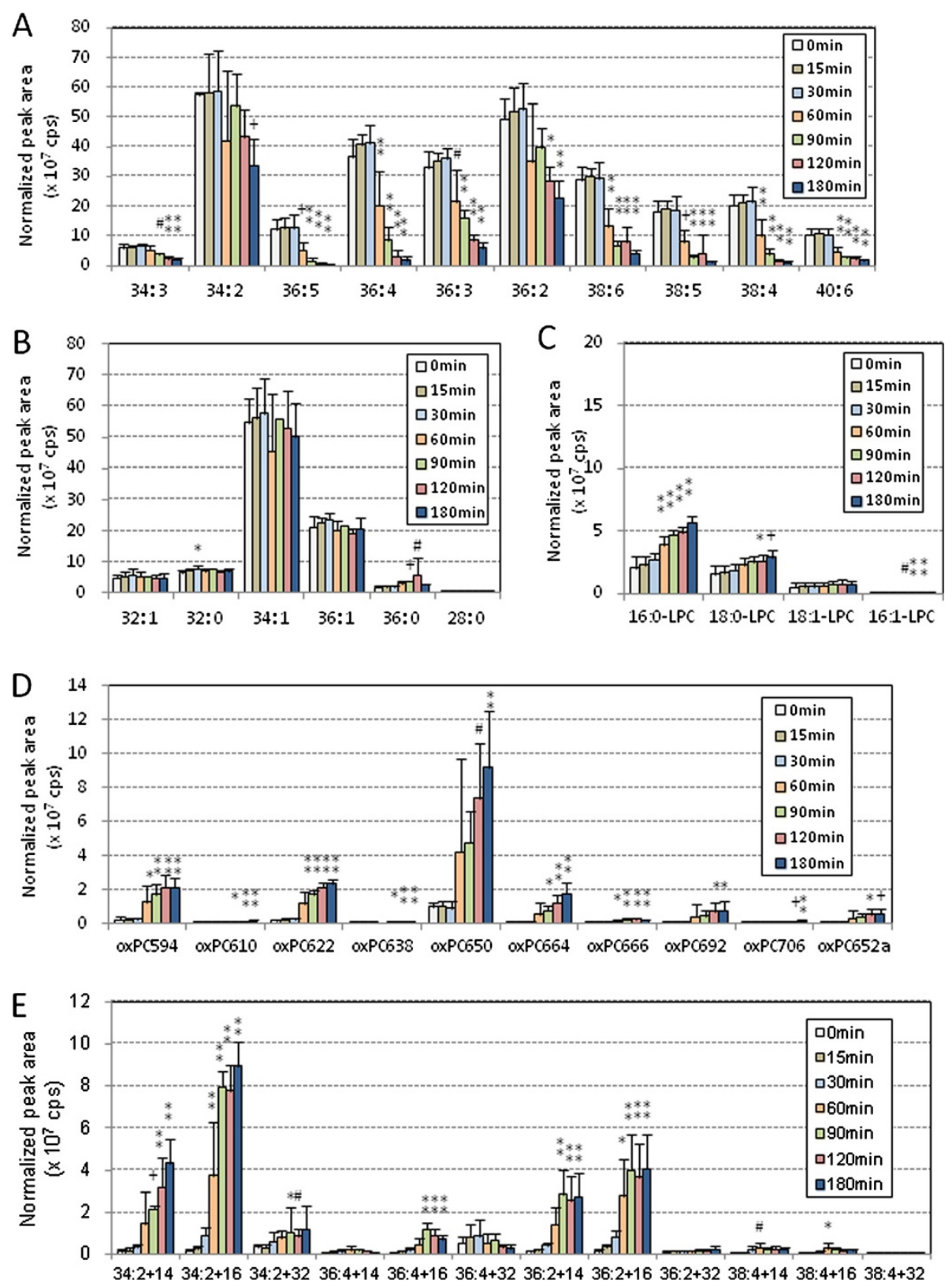

Figure 3 PC profile changes during copper-induced oxidation of human LDL treated with a PAF-AH inhibitor. Human LDL was pretreated with pefabloc, a PAF-AH inhibitor, and the treated LDL $(0.2 \mathrm{mg} / \mathrm{mL})$ was incubated with copper sulfate $(5 \mu \mathrm{M})$ at $37^{\circ} \mathrm{C}$ for up to $3 \mathrm{~h}$. Lipid extraction and LC-MS/MS analysis were performed as in Figure 2. The peak area (cps) for each species was normalized based on the peak areas of internal standard, and means and SD were calculated from six independent preparations. The 42 PC species were classified into five groups: PUFA-PC (A), S/MUFA-PC (B), lysoPC (C), cleaved-chain oxPC (D) and long-chain oxPC (E). Statistical significance of comparison with the sample without incubation (0 min) was calculated by ANOVA; ${ }^{*}, p<0.05 ; \#, p<0.01 ;+, p<0.005 ;{ }^{*}, p<0.001$.

declined sharply and some of them reduced largely, whereas S/MUFA-PC did not change. Long-chain oxPC with four double bonds were unstable and appeared transient. Interestingly, only a few oxPC species accumulated in fully oxidized LDL in vitro, namely PONPC $(\mathrm{m} / \mathrm{z}=650.6)$ and mono-oxygenated forms of linoleate-containing PC. It is likely that oxPC species derived from PC containing more than four double bonds are so susceptible to further modification either by chemically or enzymatically that they cannot accumulate in oxLDL.

$\mathrm{PCOOH}$, detected as long chain oxPC species with $\mathrm{m} / \mathrm{z}+32$, was present in LDL but was not the major products in oxLDL (Figures 2, 3, 4, 5, 6 and 7, and Additional file 1 for enlarged graphs). Kinoshita, et al. determined the $\mathrm{PCOOH}$ concentration in human plasma from healthy control was $160 \mathrm{nmol} / \mathrm{L}$ using HPLC with 
chemiluminescence detector [37]. PCOOH concentration increased to approximately 2-fold in plasma from patients with hyperlipidemia, however, the ratio of $\mathrm{PCOOH}$ in total PC was still $1 / 7,000$. Our data cannot directly transfer to quantitative calculations, but relatively small peaks for $\mathrm{PCOOH}$ in the human and rabbit LDLs among all the PC species (Figures 2 and 5) corresponds well to the previous study.

LysoPC appeared to be the major product in oxLDL. Its generation was strongly suppressed by pefabloc treatment of both human and rabbit LDL, suggesting that PAF-AH activity is critical for lysoPC generation in LDL. It is well known that PAF-AH can act on PC species with hydrophilic short chain acyl groups, in addition to PAF, to produce lysoPC [26]. Thus it is speculated that pefabloc treatment of LDL increases some oxPC species that are substrates of PAF-AH during oxLDL formation. The peak areas for several cleaved oxPC species further increased by the pefabloc treatment (Figures 4D and 7D). However, the major oxPC species that accumulated in pefabloc-treated LDL during oxidation was almost the same as that of oxLDL with active PAF$\mathrm{AH}$. Our observation agrees with a previous report by Davis et al., in which an oxPC profile in LDL oxidized with copper sulfate for $20 \mathrm{~h}$ was analyzed [35].

In addition to hydrolysis of cleaved oxPC species, the protective role of PAF-AH in oxLDL modification was suggested by oxPC-apoB adduct formation. Some chemically active oxPC may react with proteins to form adducts, and extensive hydrolysis of oxPC by PAF-AH is protective against apoB modification by oxPC products.

LysoPC generation was largely inhibited in pefabloctreated oxLDL, but still a very small increase in lysoPC remained. A possible explanation for this observation is non-enzymatic hydrolysis of oxPC. Choi, et al. reported that lysoPC can be generated through spontaneous deacylation of cleaved-chain oxPC products such as 1-palmityl-2-(4-hydroxy-7-oxo-5-heptenoyl)-PC [38]. Alternatively, other oxPC-hydrolyzing enzymes may distribute in part to LDL and contribute to lysoPC formation. We 

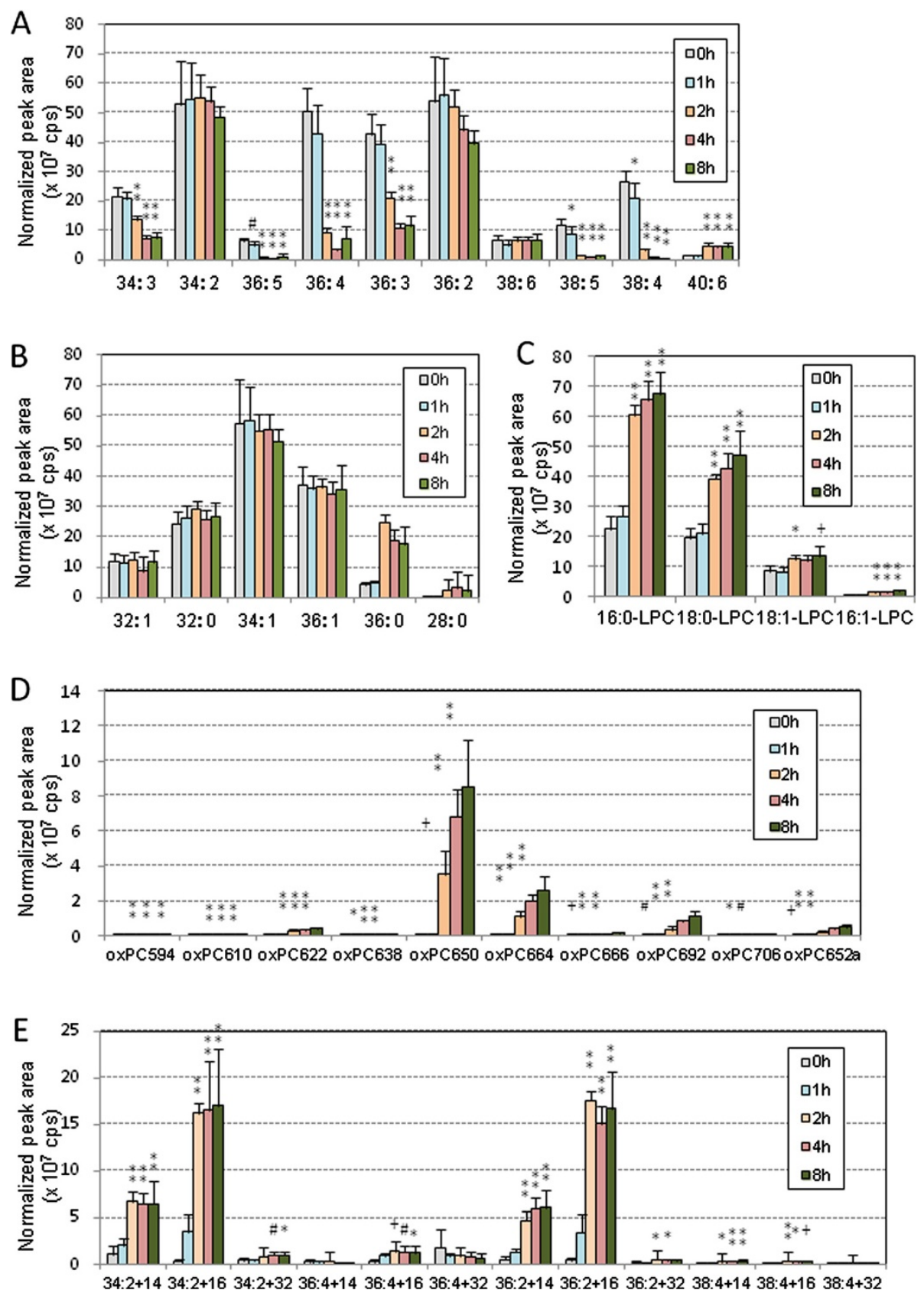

Figure 5 PC profile changes during copper-induced oxidation of rabbit LDL. Rabbit LDL $(0.1 \mathrm{mg} / \mathrm{mL})$ was incubated with copper sulfate $(5 \mu \mathrm{M})$ at $37^{\circ} \mathrm{C}$ for up to $8 \mathrm{~h}$. Lipid extraction and LC-MS/MS analysis were performed as in Figure 2. The peak area (cps) for each species was normalized based on the peak areas of internal standard, and the means and SD were calculated from six independent preparations. The 42 PC and modified PC molecular species by MRM mode, and they were classified into five categories: PUFA-PC (A), S/MUFA-PC (B), lysoPC (C), cleaved-chain $\operatorname{OxPC}(\mathrm{D})$ and long-chain $\operatorname{OxPC}(\mathrm{E})$. Statistical significance of comparison with the sample without incubation $(0 \mathrm{~h})$ was calculated by ANOVA; ${ }^{*}, p<0.05 ; \#, p<0.01 ;+, p<0.005 ;{ }^{* *}, p<0.001$.

propose that most of the oxPC species generated are not only hydrolyzed by PAF-AH but also further modified or decomposed in PAF-AH-independent manners.

Inhibition of PAF-AH in apoE-knockout mice resulted in reduction in atherosclerotic lesion size [39]. However, another study reported that adenoviral overexpression of
PAF-AH prevents injury-induced neointima in apoEknockout mice [40]. A PAF-AH inhibitor has been investigated in clinical trials and the prevention of expansion of necrotic core lesions in human was shown, however, the roles of PAF-AH in atherogenesis and oxLDL modification remain uncertain [41]. Our study suggests that 

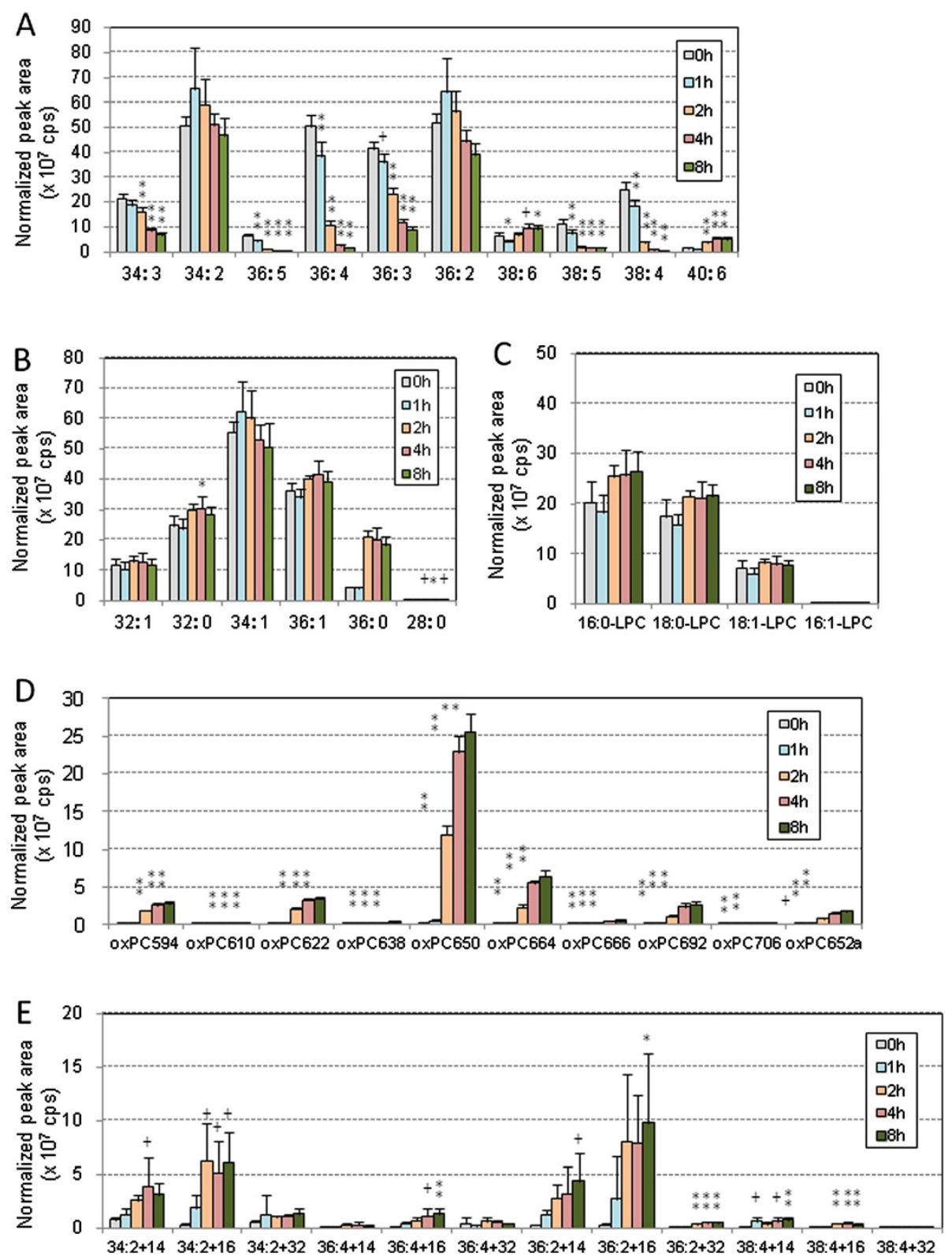

Figure 6 PC profile changes during copper-induced oxidation of rabbit LDL treated with PAF-AH inhibitor. Rabbit LDL (0.1 mg/mL) was treated with a PAF-AH inhibitor, pefabloc, and then, incubated with copper sulfate $(5 \mu \mathrm{M})$ at $37^{\circ} \mathrm{C}$ for up to $8 \mathrm{~h}$. Lipid extraction and LC-MS/MS analysis were performed as in Figure 2. The peak area (cps) for each species was normalized based on the peak areas of internal standard, and the means and SD were calculated from six independent preparations. The 42 PC species were classified into five groups: PUFA-PC (A), S/MUFA-PC (B), lysoPC (C), cleaved-chain oxPC (D) and long-chain oxPC (E). Statistical significance of comparison with the sample without incubation (0 h) was calculated by ANOVA; ${ }^{*}, p<0.05 ; \#, p<0.01 ;+, p<0.005 ;{ }^{* *}, p<0.001$.

PAF-AH inhibition decreased lysoPC formation but had little effect on oxPC accumulation. To understand the effects of PAF-AH on atherosclerosis, the pathological roles of atherogenic oxPC and lysoPC should be elucidated.

It should be noted that the current study focused on oxLDL prepared in vitro. This study is an important step toward understanding the complex nature of oxidized lipoproteins. Elucidation of the contribution of oxLDL to atherogenesis awaits further lipidomic and proteomic studies to characterize the features of circulating oxLDL.

\section{Conclusions}

We determined the PC profile of oxLDL using a lipidomic approach. The major products in copper-induced oxLDL were lysoPC species, and PAF-AH plays a critical 


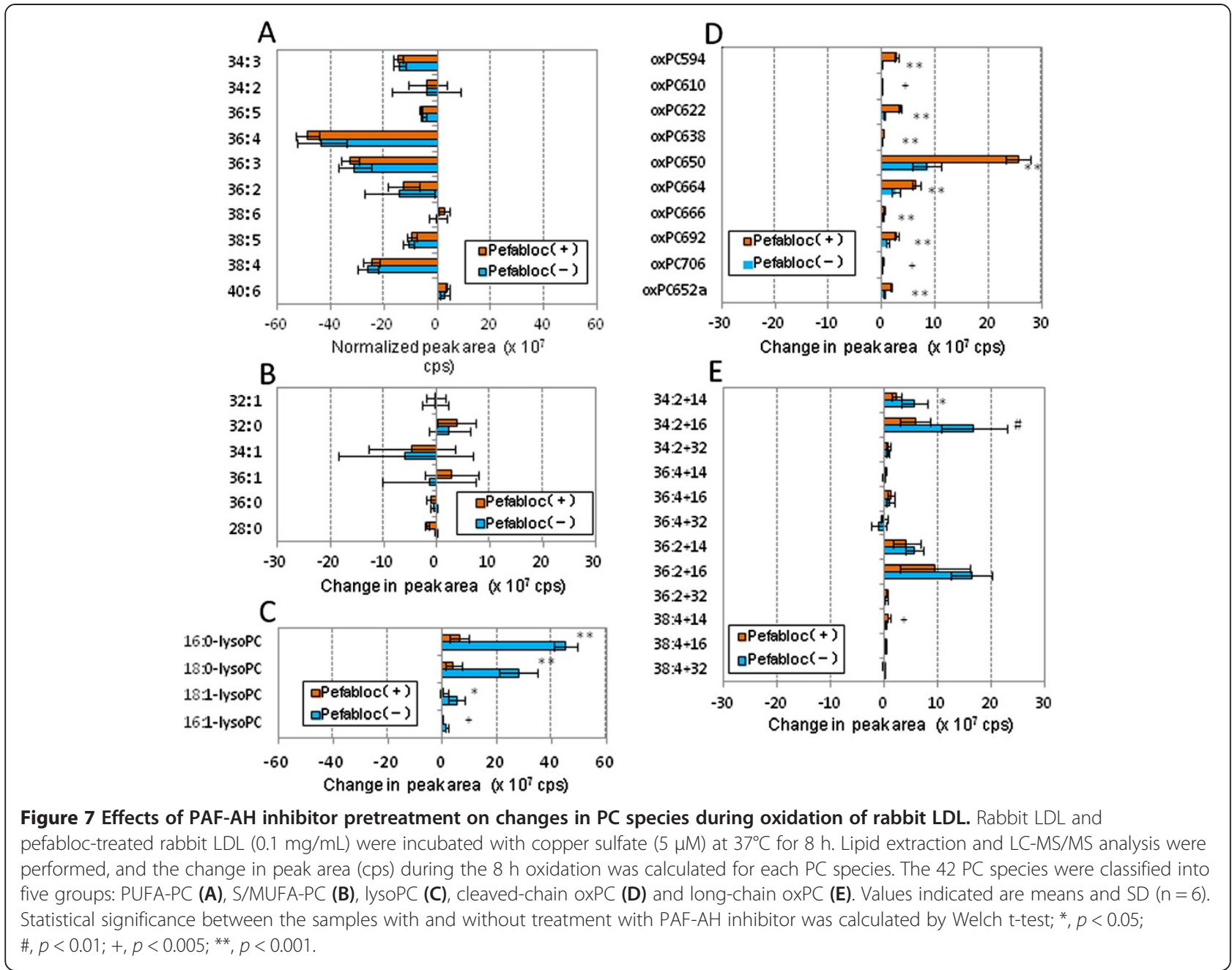

role in lysoPC generation in LDL. Although many oxPC species are generated, PONPC and long-chain oxPC species derived from linoleate-containing $\mathrm{PC}$ are the major products accumulating in oxLDL. These observations shed light on the manner in which oxPCs are modified and metabolized.

\section{Methods}

\section{Preparation and oxidation of human LDL}

Human LDL and oxLDL were prepared as described previously [9]. This study was approved by the Ethical Committee of Showa University. In brief, plasma was separated from human blood from healthy volunteers by centrifugation at 2,000 rpm for $10 \mathrm{~min}$, after which EDTA was added to the plasma (final concentration $0.25 \mathrm{mM})$. LDL was separated from the human plasma using sequential ultracentrifugation, with addition of $\mathrm{KBr}$ to adjust the density, and then dialyzed against PBS containing $0.25 \mathrm{mM}$ EDTA to remove the $\mathrm{KBr}$. The protein concentration of the LDL fraction was determined by the BCA method using BSA as standard.

To inhibit PAF-AH activity of LDL, an aliquot of the LDL fraction $(1 \mathrm{mg} / \mathrm{mL}$ ) was incubated with pefabloc (Boehringer, $0.5 \mathrm{mM})$ at $37^{\circ} \mathrm{C}$ for $30 \mathrm{~min}$ [30]. The LDL was then passed through a PD-10 desalting column (BioRad) to remove excess pefabloc. The PAF-AH activity was estimated using a PAF-AH assay kit (Cayman Chemicals).

Either LDL or pefabloc-treated LDL $(0.2 \mathrm{mg} / \mathrm{mL})$ was incubated with $\mathrm{CuSO}_{4}(5 \mu \mathrm{M})$ at $37^{\circ} \mathrm{C}$ for up to $3 \mathrm{~h}$. At the end of the incubation period, $1 \mu \mathrm{L}$ of $250 \mathrm{mM}$ EDTA was added to stop copper-induced oxidation. Oxidation reaction was evaluated by measurement of TBARS and agarose-gel electromobility assay. After mixtures of $100 \mu \mathrm{L}$ of sample and $200 \mu \mathrm{L}$ of TBA reagent $(0.375 \%$ TBA, 15\% trichloroacetic acid, and $0.25 \mathrm{M} \mathrm{HCl}$ ) were boiled for $15 \mathrm{~min}$, absorbance was measured at $535 \mathrm{~nm}$ [42]. Electromobility of oxLDL was evaluated on a $0.5 \%$ agarose gel using barbiturate buffer at $\mathrm{pH}$ 8.6. Determination of immunoreactivity to an anti-oxPC monoclonal 


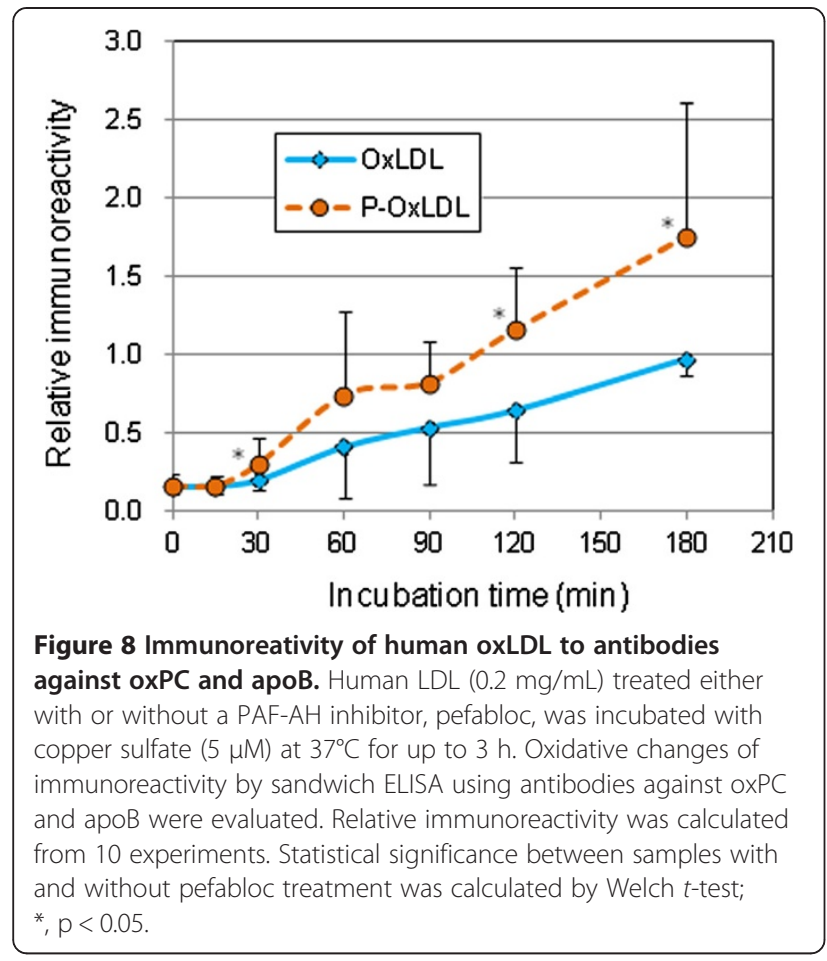

antibody and an anti-apoB polyclonal antibody was performed by sandwich ELISA as described previously [36].

\section{Preparation and oxidation of rabbit LDL}

New Zealand White rabbits (6-7 weeks old) were purchased from Kitayama Labes Co., Ltd. (Nagano, Japan). The study protocol was approved by the Animal Care Committee (permit no. 11-0221) and carried out in accordance with the "Guideline for Animal Care and Use at the Otsuka Pharmaceutical Co. Ltd.". The rabbits were acclimated and fed a cholesterol-enriched diet containing $0.5 \%$ cholesterol $(\mathrm{w} / \mathrm{w})$ in a standard rabbit chow (RC4; Oriental Yeast Co. Ltd.) at 80 g/day for 10 weeks as previously described [43]. Blood was collected from the inferior vena cava in EDTA-treated syringes under pentobarbital anesthesia. Then LDL fraction was collected from plasma by centrifugation, $0.25 \mathrm{mM}$ EDTA was added, and LDL was stored in tubes filled with argon gas in a refrigerator. After LDL was dialyzed against PBS in a refrigerator, $5 \mu \mathrm{M} \mathrm{CuSO}_{4}$ was added to $100 \mu \mathrm{g} / \mathrm{mL}$ of LDL and incubated for up to $8 \mathrm{~h}$ at $37^{\circ} \mathrm{C}$. The oxidation reaction was stopped by addition of EDTA (final $0.25 \mu \mathrm{M}$ ) to the solution. The oxLDL was stored in tubes filled with argon gas at $-80^{\circ} \mathrm{C}$.

\section{LC-MS/MS analysis of oxPC}

The profile of PC molecular species in LDL and oxLDL was determined by LC-MS/MS (AB SCIEX, QTRAP5500 equipped with Shimadzu LC-10A). LC separations were performed on an Inertsil SIL-100A column $(2.1 \times 150 \mathrm{~mm}$, GL Science, Tokyo, Japan) with elution solvent of acetonitrile/methanol/3\% formic acid $(18 / 11 / 8(\mathrm{v} / \mathrm{v} / \mathrm{v}))$ at a flow rate $200 \mu \mathrm{L} / \mathrm{min}$ as described by Morishita et al. [44]. The synthetic PC standards, didecanoyl-PC, 1-pentadecanoyl-2lysoPC, PONPC were purchased from Avanti Polar Lipid Inc. Total lipids were extracted from $10 \mu \mathrm{g}$ of oxLDL by the Bligh and Dyer method [45]. To the recovered chloroform phase, didecanoyl-PC (20 pmol) was added as internal standard. The samples were dried under $\mathrm{N}_{2}$ gas and the dissolved in $200 \mu \mathrm{L}$ of the elution solvent. For each analysis, $10 \mu \mathrm{L}$ of the sample was injected. PC species were detected and quantified using MRM procedure, in which 42 selected ions releasing a fragment ion of $\mathrm{m} / \mathrm{z}=184$ corresponding to a phosphorylcholine group were detected.

\section{Statistics}

Data are presented as means \pm standard deviation (SD). For the statistical analysis, one-way-ANOVA was carried out, and a $p$ value $<0.05$ was taken to be significant.

\section{Additional file}

Additional file 1: Enlarged figures for PC profile changes during
copper-induced oxidation of human and rabbit LDL. Some bar
graphs of the PC species shown in the Figures $2,3,5$ and 6 were too
small to see (less than $\left.1 \times 10^{7} \mathrm{cps}\right)$, this file shows the detail of the PC
profile changes of the minor species. The time course changes of PC
species during oxidation of human LDL with or without pefabloc
pretreatment (A, B) are shown in an enlarged scale. The time course
changes of ten PC species during oxidation of rabbit LDL with or without
pefabloc (C, D) are shown in an enlarged scale. Statistical significance of
comparison with the sample without incubation $(0$ min) was calculated
by ANOVA; ${ }^{*}, p<0.05 ; \#, p<0.01 ;+, p<0.005 ;{ }^{* *}, p<0.001$.

\section{Abbreviations}

apoB: Apolipoprotien B; cleaved oxPC: OxPC with truncated acyl chains; ELISA: Enzyme-linked immunosorbent assay; HDL: High-density lipoprotein; LC-MS/MS: Liquid chromatography-tandem mass spectrometry; LDL: Lowdensity lipoprotein; long-chain OxPC: OxPC with oxidized acyl chains; lysoPC: Lysophosphatidylcholine; MRM: Multiple-reaction monitoring; MUFA: Monounsaturated fatty acids; OxLDL: Oxidized low-density lipoprotein; OxPC: Oxidized phosphatidylcholine; PAF-AH: Platelet-activating factor acetylhydrolase; PC: Phosphatidylcholine; PCOOH: PC hydroperoxide; PONPC: 1-palmitoyl-2-(9-oxononanoyl) PC; PUFA: Polyunsaturated fatty acids; PUFA-PC: PC with polyunsaturated fatty acids; SD: Standard deviation; S/MUFA-PC: PC with saturated or monounsaturated fatty acids; TBARS: Thiobarbituric acid-reactive substances.

\section{Competing interests}

The authors declare that they have no competing interest.

\section{Authors' contribution}

NS, YK and HI designed the study. NS, YK, TO, NI, YM, YI, TA, RK, ToYa, HK, and $\mathrm{SI}$ performed the experiments and NS, YK and $\mathrm{HI}$ analyzed the data. $\mathrm{HI}$ and YK wrote the manuscript. ToYa, AM, SH and ToYo performed critical reading of the manuscript and discussion. All authors read and approved the final manuscript.

\section{Acknowledgements}

We thank Drs. Junken Aoki and Aska Inoue in Tohoku University for valuable suggestions and help for lipidomic analysis. This work was supported in part by grants-in-aid from the Ministry of Education, Technology, Sports, Sciences 
and Technology of Japan (MEXT), grants for the Private University High Technology Research Center Project from MEXT, and Research on Publicly Essential Drugs and Medical Devices from Japan Health Sciences Foundation. We thank Crimson Interactive Pvt. Inc. for editing service of English expressions.

\section{Author details}

${ }^{1}$ Division of Biological Chemistry, Department of Molecular Biology, Showa University School of Pharmacy, Tokyo, Japan. ${ }^{2}$ Free Radical Research Project, Otsuka Pharmaceutical Co. Ltd., Tokushima, Japan. ${ }^{3}$ Division of Health Chemistry, Department of Healthcare and Regulatory Sciences, Showa University School of Pharmacy, Tokyo, Japan. ${ }^{4}$ Department of Biochemistry, Showa University School of Medicine, Tokyo, Japan.

Received: 8 February 2014 Accepted: 6 March 2014

Published: 14 March 2014

\section{References}

1. Itabe H: Oxidized low density lipoprotein: what is understood and what remains to be clarified. Biol Pharm Bull 2003, 26:1-9.

2. Igarashi $Y, O k a$ Y, Katagiri H: Circulating oxidized LDL: a biomarker and a pathological factor. Cur Opin Lipidol 2009, 20:363-369.

3. Witztum JL, Steinberg D: Role of oxidized low density lipoprotein in atherogenesis. J Clin Invest 1991, 91:1785-1792

4. Ehara S, Ueda M, Naruko T, Haze K, Itoh A, Otsuka M, Komatsu R, Matsuo T, Itabe H, Takano T, Tsukamoto Y, Yoshiyama M, Takeuchi K, Yoshikawa J, Becker AE: Elevated levels of oxidized low density lipoprotein show a positive relationship with the severity of acute coronary syndromes. Circulation 1955-1960, 2001:103.

5. Holvoet P, Van Cleemput J, Collen D, Vanhaecke J: Oxidized low density lipoprotein is a prognostic marker of transplant-associated coronary artery disease. Arterioscler Thromb Vasc Biol 2000, 20:698-702.

6. Tsimikas S, Bergmark C, Beyer RW, Patel R, Pattison J, Miller E, Juliano J, Witztum JL: Temporal increases in plasma markers of oxidized lowdensity lipoprotein strongly reflect the presence of acute coronary syndromes. J Am Coll Cardiol 2003, 41:360-730.

7. Naruko T, Ueda M, Ehara S, Itoh A, Haze K, Shirai N, Ikura Y, Ohsawa M, Itabe H, Kobayashi Y, Yamagishi H, Yoshiyama M, Yoshikawa J, Becker AE: Persistent high levels of plasma oxidized low-density lipoprotein after acute myocardial infarction predict stent restenosis. Arterioscler Thromb Vasc Biol 2006, 26:877-883.

8. Itabe H, Ueda M: Measurement of plasma oxidized low-density lipoprotein and its clinical implications. J Atheroscler Thromb 2007, 14:1-11.

9. Itabe H, Yamamoto H, Suzuki M, Kawai Y, Nakagawa Y, Suzuki A, Imanaka T, Takano T: Oxidized phosphatidylcholines that modify proteins. Analysis by monoclonal antibody against oxidized low density lipoprotein. J Biol Chem 1996, 271:33208-33217.

10. Heinecke JW: Oxidants and antioxidants in the pathogenesis of atherosclerosis: implications for the oxidized low density lipoproteins hypothesis. Atherosclerosis 1998, 141:1-15.

11. Obama T, Kato R, Masuda Y, Takahashi K, Aiuchi T, Itabe H: Analysis of modified apolipoprotein B-100 structures formed in oxidized low-density lipoprotein using LC-MS/MS. Proteomics 2007, 7:2132-2141.

12. Podrez EA, Poliakov E, Shen Z, Zhang R, Deng Y, Sun M, Finton PJ, Shan L, Gugiu B, Fox PL, Hoff HF, Salomon RG, Hazen SL: Identification of a novel family of oxidized phospholipids that serve as ligands for the macrophage scavenger receptor CD36. J Biol Chem 2002, 277:38503-38516.

13. Hörkkö S, Bird DA, Miller E, Itabe H, Leitinger N, Subbanagounder G, Berliner $J A$, Friedman P, Dennis EA, Curtiss LK, Palinski W, Witztum JL: Monoclonal autoantibodies specific for oxidized phospholipids or oxidized phospholipid-protein adducts inhibit macrophage uptake of oxidized low-density lipoproteins. J Clin Invest 1999, 103:117-128,

14. Davies SS, Pontsler AV, Marathe GK, Harrison KA, Murphy RC, Hinshaw JC, Prestwich GD, Hilaire AS, Prescott SM, Zimmerman GA, McIntyre TM: Oxidized alkyl phospholipids are specific, high affinity peroxisome proliferator-activated receptor $\gamma$ ligands and agonists. J Biol Chem 2001, 276:16015-16023.

15. Watson AD, Leitinger N, Navab M, Faull KF, Hörkkö S, Witztum JL, Palinski W, Schwenke D, Salomon RG, Sha W, Subbanagounder G, Fogelman AM, Berliner JA: Structural identification by mass spectrometry of oxidized phospholipids in minimally oxidized low density lipoprotein that induce monocyte/endothelial interactions and evidence for their presence in vivo. J Biol Chem 1997, 272:13597-13607.

16. Lee H, Shi W, Tontonoz P, Wang S, Subbanagounder G, Hedrick CC, Hama S, Borromeo C, Evans RM, Berliner JA, Nagy L: Role for peroxisome proliferator-activated receptor a in oxidized phospholipid-induced synthesis of monocyte chemotactic protein-1 and interleukin- 8 by endothelial cells. Circ Res 2000, 87:516-521.

17. Parhami F, Morrow AD, Balucan J, Leitinger $N$, Watson AD, Tintut $Y$, Berliner $J A$, Demer LL: Lipid oxidation products have opposite effects on calcifying vascular cell and bone cell differentiation. A possible explanation for the paradox of arterial calcification in osteoporotic patients. Arterioscler Thromb Vasc Biol 1997, 17:680-687.

18. Hazen SL: Oxidized phospholipids as endogenous pattern recognition ligands in innate immunity. J Biol Chem 2008, 283:15527-15531.

19. Asai A, Okajima F, Nakagawa K, Ibusuki D, Tanimura K, Nakajima Y, Nagao M, Sudo M, Harada T, Miyazawa T, Oikawa S: Phosphatidylcholine hydroperoxide-induced THP-1 cell adhesion to intracellular adhesion molecule-1. J Lipid Res 2009, 50:957-965.

20. Sugiyama S, Kugiyama K, Ohgushi M, Fujimoto K, Yasue H: Lysophosphatidylcholine in oxidized low-density lipoprotein increases endothelial susceptibility to polymorphonuclear leukocyte-induced endothelial dysfunction in porcine coronary arteries: role of protein kinase C. Circ Res 1994, 74:565-575.

21. Chai Y-C, Binion DG, Macklis R, Chisolm GM: Smooth muscle cell proliferation induced by oxidized LDL-borne lysophosphatidylcholine: Evidence for FGF-2 release from cells not extracellular matrix. Vasc Pharmacol 2002, 38:229-237.

22. Imai H, Nakagawa Y: Biological significance of phospholipid hydroperoxide glutathione peroxidase (PHGPx, GPx4) in mammalian cells. Free Radic Biol Med 2003, 34:145-169.

23. Itabe $\mathrm{H}$, Kudo I, Inoue K: Preferential hydrolysis of oxidized phospholipids by peritoneal fluid of rats treated with casein. Biochim Biophys Acta 1988, 963:192-200.

24. Itabe H, Hosoya R, Karasawa K, Jimi S, Saku K, Takebayashi S, Imanaka T, Takano T: Metabolism of oxidized phosphatidylcholines formed in oxidized low density lipoprotein by lecithin-cholesterol acyltransferase. J Biochem 1999, 126:153-161.

25. Stafforini DM, Prescott SM, Mclntyre TM: Human plasma platelet-activating factor acetylhydrolase. Purification and properties. J Biol Chem 1987, 262:4223-4230.

26. Smiley PL, Stremler KE, Prescott SM, Zimmerman GA, Mclntyre TM: Oxidatively fragmented phosphatidylcholines activate human neutrophils through the receptor for platelet-activating factor. J Biol Chem 1991, 266:11104-11110.

27. Steinbrecher UP, Pritchard PH: Hydrolysis of phosphatidylcholine during LDL oxidation is mediated by platelet-activating factor acetylhydrolase. J Lipid Res 1989, 30:305-315.

28. Maki N, Hoffman DR, Johnston JM: Platelet-activating factor acetylhydrolase activity in maternal, fetal, and newborn rabbit plasma during pregnancy and lactation. Proc Natl Acad Sci U S A 1988, 88:728-732.

29. Noto H, Hara M, Karasawa K, Iso-O N, Satoh H, Togo M, Hashimoto Y, Yamada Y Kosaka T, Kawamura M, Kimura S, Tsukamoto K: Human plasma plateletactivating factor acetylhydrolase binds to all the murine lipoproteins, conferring protection against oxidative stress. Arterioscler Thromb Vasc Biol 2003, 23:829-835.

30. Dentan C, Tselepis AD, Chapman MJ, Ninio E: Pefabloc, 4-[2-aminoethyl] benzenesulfonyl fluoride, is a new, potent nontoxic and irreversible inhibitor of PAF-degrading acetylhydrolase. Biochim Biophys Acta 1996, 1299:353-357.

31. Ahmed Z, Ravandi A, Maguire GF, Emili A, Draganov D: Multiple Substrates for paraoxonase-1 during oxidation of phosphatidylcholine by peroxynitrite. Biochem Biophys Res Commun 2002, 290:391-396.

32. Goyal J, Wang K, Liu M, Subbaiah PV: Novel function of lecithin-cholesterol acyltransferase: Hydrolysis of oxidized polar phospholipids generated during lipoprotein oxidation. J Biol Chem 1997, 272:16231-16239.

33. Spickett CM, Reis A, Pitt AR: Identification of oxidized phospholipids by electrospray ionization mass spectrometry and LC-MS using a QQLIT instrument. Free Radic Biol Med 2011, 51:2133-2149.

34. Houjou T, Yamatani K, Nakanishi H, Imagawa M, Shimizu T, Taguchi R: Rapid and selective identification of molecular species in phosphatidylcholine and sphingomyelin by conditional neutral loss scanning and $\mathrm{MS}^{3}$. Rapid Commun Mass Spectrom 2004, 18:3123-3130. 
35. Davis B, Koster G, Douet LJ, Scigelova M, Woffendin G, Ward JM, Smith A, Humphries J, Burnand KG, Macphee CH, Postle AD: Electrospray ionization mass spectrometry identifies substrates and products of lipoproteinassociated phospholipase $A_{2}$ in oxidized human low density lipoprotein. J Biol Chem 2008, 283:6428-37.

36. Itabe H, Yamamoto H, Imanaka T, Shimamura K, Uchiyama H, Kimura J, Sanaka T, Hata Y, Takano T: Sensitive detection of oxidatively modified low density lipoprotein using a monoclonal antibody. J Lipid Res 1996, 37:45-53.

37. Kinoshita M, Oikawa S, Hayasaka K, Sekikawa A, Nagashima T, Toyota T, Miyazawa T: Age-related increase in plasma phosphatidylcholine hydroperoxide concentration in control subjects and patients with hyperlipidemia. Clin Chem 2000, 46:822-828.

38. Choi J, Zhang W, Gu X, Chen X, Hong L, Laird JM, Salomon RG: Lysophosphatidylcholine is generated by spontaneous deacylation of oxidized phospholipids. Chem Res Toxicol 2010, 24:111-118.

39. Wilensky RL, Shi Y, Mohler ER 3rd, Hamamdzic D, Burgert ME, Li J, Postle A, Fenning RS, Bollinger JG, Hoffman BE, Pelchovitz DJ, Yang J, Mirabile RC, Webb CL, Zhang L, Zhang P, Gelb MH, Walker MC, Zalewski A, Macphee CH: Inhibition of lipoprotein-associated phospholipase $A_{2}$ reduces complex coronary atherosclerotic plaque development. Nat Med 2008, 14:1059-1066

40. Quarck R, De Geest B, Stengel D, Mertens A, Lox M, Theilmeier G, Michiels C, Raes M, Bult H, Collen D, Van Veldhoven P, Ninio E, Holvoet P: Adenovirusmediated gene transfer of human platelet-activating factoracetylhydrolase prevents injury-induced neointima formation and reduces spontaneous atherosclerosis in apolipoprotein E-deficient mice. Circulation 2001, 103:2495-2500.

41. Rosenson RS, Stafforini DM: Modulation of oxidative stress, inflammation, and atherosclerosis by lipoprotein-associated phospholipase $A_{2}$. J Lipid Res 2012, 53:1767-1782.

42. Beuge JA, Aust SD: Microsomal lipid peroxidation. Methods Enzymol 1978, 52:302-310.

43. Niimi M, Keyamura Y, Nozako M, Koyama T, Kohashi M, Yasufuku R, Yoshikawa T, Fan J: Pobucol inhibits the initiation of atherosclerosis in cholesterol-fed rabbits. Lipids Health Dis 2013, 12:166.

44. Morishita K, Aiboshi J, Kobayashi T, Mikami S, Yokoyama Y, Ogawa K, Yokota $\mathrm{H}$, Otomo $\mathrm{Y}$ : Lipidomics analysis of mesenteric lymph after trauma and hemorrhagic shock. J Trauma Acute Care Surg 2012, 72:1541-1547.

45. Bligh EA, Dyer WJ: A rapid method to total lipid extraction and purification. Can J Biochem Physiol 1959, 37:911-917.

doi:10.1186/1476-511X-13-48

Cite this article as: Sasabe et al: Time course-changes in

phosphatidylcholine profile during oxidative modification of

low-density lipoprotein. Lipids in Health and Disease 2014 13:48.

\section{Submit your next manuscript to BioMed Central and take full advantage of:}

- Convenient online submission

- Thorough peer review

- No space constraints or color figure charges

- Immediate publication on acceptance

- Inclusion in PubMed, CAS, Scopus and Google Scholar

- Research which is freely available for redistribution 